\title{
Laser-assisted head and neck surgery in the COVID-19 pandemic: controversial evidence and precautions
}

\author{
Oreste Gallo, $\mathrm{MD}^{1}$ \\ ${ }^{1}$ Careggi University Hospital
}

May 13, 2020

Oreste Gallo, MD; Luca Giovanni Locatello, MD

Department of Otorhinolaryngology,

Careggi University Hospital, Florence - Largo Brambilla, 3 - 50134 Florence, Italy

* Corresponding author: Prof. Oreste Gallo, MD, Department of Otorhinolaryngology, Careggi University Hospital, Florence - Largo Brambilla, 3 - 50134 Firenze, Italy. +39 0557947989, oreste.gallo at unifi.it

Keywords: COVID-19, laser-assisted surgery, surgical plume, prevention, surgical safety

Authors' contributions: Gallo: Conceptualization, supervision and writing - review and editing; Locatello: Conceptualization, resources, supervision, and writing - review and editing.

Conflict of Interest: all authors declare they have nothing to disclose.

This research did not receive any specific grant from funding agencies in the public, commercial, or not-forprofit sectors.

Many of the safety issues related to the novel COVID-19 in our routine surgical practice were thoroughly presented in this Journal. [1] However, the authors did not discuss an important field of head and neck surgery: laser-assisted procedures. Historically, human-papillomavirus (HPV) in the setting of respiratory recurrent papillomatosis is the prototype of the biological hazards of the laser-generated surgical plume.[2] In the past, it was shown that surgical vaporization was capable to contaminate the staff's personal protective equipment (PPE) with viable and infectious HPV virions. [2] Despite the growing evidence documenting a key role of high-risk HPV infections in the pathogenesis and development of head and neck cancer, the risk assessment of potential viral infection after exposure to laser plume is still controversial. [3] The biohazard might not be limited to HPV airborne transmission, but also other bacteria and viruses, including (possibly) SARS-CoV-2. For instance, Kwak et al. documented Hepatitis B (HBV) DNA in surgical smoke from 10 out of $11 \mathrm{HBV}+$ patients undergoing robotic laparoscopic surgery thus suggesting a potential risk of airborne HBV infection.[4] Heat-generating procedures by electrosurgical equipment or lasers can induce thermal disruption of viable human cells and they are able to aerosolize hazardous particles. The thermal effect of lasers on biological tissues is a complex process resulting from the conversion of light to heat whose effects depend upon several factors: the physical denaturation and/or destruction is a function of laser settings (wavelength, power, time and mode of emission, beam profile, and spot size) and the target of the procedure (thermal parameters, optical coefficient, etc.).[5,6] During ablative surgery, the tissue is heated by the absorbed laser energy and it evaporates or sublimates, while, at higher power, the tissue is typically converted to plasma. This means that during laser-tissue interaction aerosolized blood and interstitial and intracellular fluids, along with their possible burden of viral pathogens and hazardous chemicals are forcefully ejected in the operating room.[6] Nonetheless, controversies exist in the literature regarding efficient viral infection of healthcare staff after exposure to surgical smoke. $[3,5,6]$ 
Surgical use of different types of lasers $\left(\mathrm{CO}_{2}, \mathrm{Nd}\right.$ :YAG, KTP...) is common not only in the head and neck but also in gynecology, dermatology, and respiratory medicine among other fields. Even though there is a lack of conclusive data on plume-borne contamination, there is an urgent need to raise awareness of its risks during the COVID-19 pandemic. In the next years, our daily practice of transoral laser-assisted surgery, an incontournable strategy to treat several benign and malignant lesions of the upper aerodigestive tract with excellent oncological and functional results, is going to be deeply modified. High viral loads, especially in the nose and the pharynx, can be detected after symptom onset but general consensus exists on SARS-CoV-2 diffusion by droplet transmission even from asymptomatic individuals, therefore it is conceivable that every laser procedure is to be considered as high-risk.[7]

While waiting for more robust specific evidence, we would like to recall some precautionary measures, inspired by the most recent literature,[8] that ought to be implemented for all laser-assisted procedures:

1. Always discuss alternative therapeutic strategies in a multidisciplinary team and postpone laser therapy if it is not urgent;

2. Perform RT-PCR test for detection of SARS-CoV-2 RNA before every procedure;

3. During routine preoperative exams, non-enhanced chest computed tomography is reported to have a higher sensitivity for COVID-19 detection than RT-PCR;

4. For small and easily accessible lesions, resection by cold instruments should be preferred;

5. Laser surgery should be performed in an operating room with a highly efficient negative-pressure system;

6. Sterilize laser handpieces after use and frequently change surgical gloves, especially after direct contact with the instrument;

8. All the staff should wear highly protective PPE, including goggles and gloves and highly protective masks (i.e., N95) with gas adsorption filters;

9. Disposable double plume evacuation systems with filters that remove particulates up to 0.1 microns (the so-called ULPA, ultra-low particulate air filters) should be available;

10. Reduce the presence in the theater of all the unnecessary personnel and perform adequate training for all staff members to enhance awareness about the hazards of the surgical smoke in the COVID-19 outbreak.

In this evolving context, head and neck laser-assisted surgery must be in all cases considered a high-risk aerosol-generating procedure and the highest attention must be paid to surgical safety until evidence-based protocols are available.

\section{Bibliography}

1. Panuganti BA, Pang J, Califano J, Chan JYK. Procedural precautions and personal protective equipment during head and neck instrumentation in the COVID-19 era. Head Neck; April 2020; https://doi.org/10.1002/hed.26220

2. Ilmarinen T, Auvinen E, Hiltunen-Back E, Ranki A, Aaltonen L M, Pitkäranta A..Transmission of human papillomavirus DNA from patient to surgical masks, gloves and oral mucosa of medical personnel during treatment of laryngeal papillomas and genital warts. European Archives of Oto-RhinoLaryngology. 2012; 269(11): 2367-2371.

3. Manson LT, Damrose EJ. Does exposure to laser plume place the surgeon at high risk for acquiring clinical human papillomavirus infection? Laryngoscope. 2013; 123(6), 1319-20

4. Kwak HD, Kim SH, Seo YS, Song KJ. Detecting hepatitis B virus in surgical smoke emitted during laparoscopic surgery. Occup Environ Med. 2016;73, 857-63.

5. Alp E, Bijl D, Bleichrodt RP, Hansson B, Voss A. Surgical smoke and infection control. J Hosp Infect. $2006 ; 62,1-5$

6. Smalley PJ. Laser safety: risks, hazards, and control measures.Laser Ther. 2011; 20 (2), 95-106.

7. Zou L, Ruan F, Huang M, Liang L, Huang H, Hong Z, Yu J, Kang M, Song Y, Xia J, Guo Q, Song T, He J, Yen HL, Peiris M, Wu J. SARS-CoV-2 Viral Load in Upper Respiratory Specimens of Infected 
Patients.N Engl J Med. 2020 Feb 19.https://doi.org/10.1056/NEJMc2001737.

8. Liu, Y., Song, Y., Hu, X., Yan, L., \& Zhu, X. (2019). Awareness of surgical smoke hazards and enhancement of surgical smoke prevention among the gynecologists. Journal of Cancer, 10(12), 2788. 